\title{
Phase-sensitive Optical Time Domain Reflectometer Assisted by First-order Raman Amplification for Distributed Vibration Sensing Over $>100 \mathrm{~km}$
}

\author{
Hugo F. Martins, Sonia Martín-López, Pedro Corredera, Massimo L. Filograno, Orlando Frazão, \\ and Miguel Gonzalez-Herráez, Senior Member, OSA
}

\begin{abstract}
In this study, the authors present an experimental and theoretical description of the use of first order Raman amplification to improve the performance of a Phase-sensitive optical time domain reflectometer ( $\phi$ OTDR) when used for vibration measurements over very long distances. A special emphasis is given to the noise which is carefully characterized and minimized along the setup. A semiconductor optical amplifier and an optical switch are used to greatly decrease the intra-band coherent noise of the setup and balanced detection is used to minimize the effects of RIN transferred from the Raman pumps. The sensor was able to detect vibrations of up to $250 \mathrm{~Hz}$ (close to the limits set by the time of flight of light pulses) with a resolution of $10 \mathrm{~m}$ in a range of $125 \mathrm{~km}$. To achieve the above performance, no post-processing was required in the $\phi$ OTDR signal. The evolution of the $\phi$ OTDR signal along the fiber is also shown to have a good agreement with the theoretical model.
\end{abstract}

Index Terms-Distributed sensor, Raman scattering, optical fiber sensors, phase-sensitive optical time domain reflectometry (OTDR), vibration sensor.

\section{INTRODUCTION}

$\mathbf{T}$ HE interest in fiber optic sensors has increased significantly over the last decade due to their intrinsic properties, such as immunity to electromagnetic noise, small size, geometric versatility, lightweight, relatively low cost, possibility of remote operation and multiplexing capability. In most systems, the fiber can be used as both the sensing element and the communication channel, which allows numerous convenient solutions

Manuscript received November 13, 2013; revised February 18, 2014; accepted February 21, 2014. Date of publication February 24, 2014; date of current version March 17, 2014. This work was supported in part by the European Research Council through Starting Grant U-FINE under Grant 307441, in part by the Spanish "Plan Nacional de I+D+i" under Projects TEC201237958-C02-01 and TEC2012-37958-C02-02, in part by the regional program FACTOTEM2 funded by the Comunidad de Madrid and the INTERREG SUDOE program ECOAL-MGT. The work of H. F. Martins was supported by the Portuguese Fundação para a Ciência e Tecnologia for providing his $\mathrm{PhD}$ Scholarship, SFRH/BD/76991 / 2011

H. F. Martins and O. Frazão are with the Faculdade de Ciências da Universidade do Porto, 4169007 Porto, Portugal, and also with INESC TEC, 4169-007 Porto, Portugal (e-mail: hfm@inescporto.pt; ofrazao@fc.up.pt).

S. Martin-Lopez and M. Gonzalez-Herraez are with the Departamento de Electrónica, Universidad de Alcalá, 28871 Madrid, Spain (e-mail: sonia.martin@depeca.uah.es; miguel.gonzalezh@uah.es).

P. Corredera and M. L. Filograno are with the Instituto de Óptica, 28006 Madrid, Spain (e-mail: p.corredera@csic.es; m.f@csic.es).

Color versions of one or more of the figures in this paper are available online at http://ieeexplore.ieee.org.

Digital Object Identifier 10.1109/JLT.2014.2308354 using this type of technology. Distributed sensors represent a particular category of fiber optic sensors which allows for the monitoring of different physical parameters (strain, temperature, vibration, pressure, etc.) at any point along a fiber. Distributed sensors present clear advantages over conventional point sensors when the number of points to be monitored is large, due to their low cost per monitored point [1].

AMONG the distributed sensing techniques, phase-sensitive optical time domain reflectometry ( $\phi$ OTDR) is a powerful technique that allows the fully distributed monitoring of vibrations along an optical fiber cable. There is a wide literature on experimental realizations of $\phi$ OTDR systems to detect vibrations [2]-[7]. Unlike traditional OTDR, which uses incoherent light and therefore can only measure intensity variations along the fiber, in $\phi$ OTDR operation a pulse of highly coherent light is used. A $\phi$ OTDR is therefore sensitive to the relative phases of the reflected fields coming from the different scattering centers around a certain position of the fiber.

Given its potential for distributed measurement of vibrations, $\phi$ OTDR presents an attractive solution for intrusion monitoring over large perimeters and has therefore attracted considerable attention for more than twenty years [2]. Conventional $\phi$ OTDR sensors allow the distributed measurement of vibrations over dynamic ranges of a few tens of kilometers [3], with spatial resolutions in the range of meters. In field tests, $\phi$ OTDR systems have been demonstrated to have enough sensitivity to detect people walking on top of a buried fiber [4]. Distributed vibration measurements up to $40 \mathrm{kHz}$ with a resolution of $5 \mathrm{~m}$ were also demonstrated in [5], which could be used in vibration-based structural damage identification or monitoring [8]. Temperature and strain measurements have also been demonstrated by analyzing the cross-correlation of $\phi \mathrm{OTDR}$ traces with different input wavelength pulses [6].

In $\phi$ OTDR sensors, having the best possible range and resolution are generally intended features. Moreover, a good visibility and high signal-to-noise ratio (SNR) in the $\phi$ OTDR trace are required in order to ensure reliable vibration measurements. If the coherence of the $\phi \mathrm{OTDR}$ pulse remains high along the fiber (with respect to the pulse width) then the visibility along the trace will remain high. As for the range, resolution and SNR, these are tightly related parameters. Generally, the increase of the input $\phi$ OTDR pulse peak power will increase the dynamic range and SNR of a $\phi \mathrm{OTDR}$, however this approach is limited due to the onset of nonlinear effects [7]. Optical amplification [9] 
or signal post-processing [10] is therefore required in order to increase the sensing range.

Raman amplification has been used for more than two decades to increase the performance of optical communication systems [11]-[13] and since then, it has been widely described theoretically and experimentally in the literature for a number of long-distance applications [14]-[21]. In optical sensing, Raman amplification has been implemented as a very attractive solution to increase the sensing range of distributed [15], [16] and point [17] fiber sensors. By exploiting the virtual transparency created by second-order Raman pumping in optical fibers [18], a new method to extend the range of Brillouin optical time domain analysis (BOTDA) systems was also proposed and demonstrated [19]. One of the main concerns of the use of this technique is the noise introduced by the Raman pumps, mainly due to relative intensity noise (RIN) transfer from the Raman pumps to the signal [20], [21].

In $\phi$ OTDR operation, Raman amplification can be used to maintain the $\phi$ OTDR pulse power high along the whole fiber length, thus extending the sensing range. However, to our knowledge, the use of Raman amplification to assist $\phi$ OTDR operation is not well documented. Some encouraging previous efforts have been reported to use first order Raman amplification in order to measure $\phi \mathrm{OTDR}$ traces of up to $74 \mathrm{~km}$ [9]. In this case however, the performance as vibration sensor was not clearly established, as no vibration measurement was performed and no in-depth study of signal noise was developed.

Along with this study, we present a theoretical and experimental description of the use of first order Raman amplification to improve the performance of a $\phi \mathrm{OTDR}$ when used for vibration measurements. The evolution of the $\phi$ OTDR signal along the fiber is recorded for different Raman pump powers showing a good agreement with the theoretical model. The main novelty in this setup comes from the use of Raman amplification combined with the noise reduction provided by the use of balanced detection. In addition, a semiconductor optical amplifier (SOA) and an optical switch are used to gate the $\phi$ OTDR pulses, achieving a strong reduction of the coherent in-band noise [5]. Special care is taken to ensure high extinction ratio (ER) $\phi$ OTDR pulses. The use of balanced detection is demonstrated to minimize the effects of the RIN transfer from the Raman pumps to the detected signal, leading to a reliable detection of vibrations up to $100 \mathrm{~km}$. Vibration measurements are successfully demonstrated with frequency sampling values close to the limits set by the time of flight of light pulses. In particular, the sensor was able to detect vibrations of up to $250 / 300 \mathrm{~Hz}$ in a distance of $125 / 110 \mathrm{~km}$ with a resolution of $10 \mathrm{~m}$. The complexity of the scheme is also kept to a minimum as no post-processing, extremely high coherence lasers or coherent detection methods are required.

\section{THEORETICAL MODEL}

In this section, we provide a mathematical model for the evolution of the Raman-assisted $\phi$ OTDR trace along the distance. This model just reflects the "average" behavior of the trace evolution, and not the statistical fluctuations expectable in any $\phi$ OTDR. For a detailed statistical analysis of these fluctuations, the interested reader is invited to read reference [5].

For the theoretical model, we assume that two similar continuous-wave (CW) Raman pumps (at $1455 \mathrm{~nm}$ ) are launched in both ends of the fiber, providing Raman gain $\left(g_{R}\right)$ at the wavelength of the $\phi$ OTDR pulse $(1550 \mathrm{~nm})$. The $\phi$ OTDR pulse is shaped from highly coherent light and its wavelength is located near the peak Raman gain of the fiber. In our analysis, the pulse is delivered at $z=0$ into the fiber and propagates in the forward direction (towards increasing values of $z$ ). As it goes thought the fiber, the Rayleigh backscattered light from the $\phi$ OTDR pulse will create a $\phi$ OTDR signal (at the same wavelength), which propagates backward until the input end of the fiber, where it is detected. The fiber is supposed to present constant losses at the wavelength of the Raman pump $\left(\alpha_{R}\right)$ and also at the $\phi$ OTDR pulse wavelength $\left(\alpha_{P}\right)$.

In normal operation of our Raman-assisted $\phi$ OTDR, the pulse peak power is about two orders of magnitude below the power of the Raman pumps. Therefore, depletion effects are neglected [16]. For the purpose of knowing the signal evolution along the fiber, our model also neglects amplified spontaneous emission (ASE) created by the Raman pumps and Double Rayleigh Backscatter. With the above assumptions, the evolution of the forward (+) and backward (-) propagating Raman pump powers along the optical fiber can be obtained by solving the equations [16]:

$$
\frac{d P_{R}^{ \pm}(z)}{d z}=\mp \alpha_{R} P_{R}^{ \pm}(z)
$$

As for the evolution of the $\phi$ OTDR pulse power, it can be obtained solving the equation:

$$
\frac{d P_{P}(z)}{d z}=-\alpha_{P} P_{P}(z)+g_{R} P_{P}(z)\left[P_{R}^{+}(z)+P_{R}^{-}(z)\right]
$$

Considering the boundary conditions given by the optical powers launched into the fiber $\left(P_{R}^{+}(0)\right.$ and $P_{R}^{-}(L)$ are the forward $(+)$ and backward (-) input Raman pump powers and $P_{P}(0)$ is the $\phi$ OTDR input peak pulse power) the evolution of the Raman pump powers and the $\phi$ OTDR pulse power along the optical fiber will be given by [16]:

$$
\begin{aligned}
P_{R}^{+}(z)= & P_{R}^{+}(0) \cdot e^{-\alpha_{R} \cdot z} \\
P_{R}^{-}(z)= & P_{R}^{-}(L) \cdot e^{\alpha_{R} \cdot(z-L)} \\
P_{P}(z)= & P_{P}(0) \cdot e^{-\alpha_{P} \cdot z} \\
& \times \exp \left\{\frac{g_{R}}{\alpha_{R}} P_{R}^{-}(L) \cdot\left[e^{\alpha_{R} \cdot(z-L)}-e^{-\alpha_{R} \cdot L}\right]\right. \\
& \left.-\frac{g_{R}}{\alpha_{R}} P_{R}^{+}(0) \cdot\left[e^{-\alpha_{R} \cdot z}-1\right]\right\} .
\end{aligned}
$$

When the $\phi$ OTDR pulse goes through a given point $\mathrm{z}_{0}$, the power of the $\phi$ OTDR signal reflected at that point $\left(P_{S}^{z}{ }^{0}\left(z_{0}\right)\right)$, will be given by:

$$
P_{S}^{z_{0}}\left(z_{0}\right)=\alpha_{\text {RayleighBackscatter }} \cdot P_{P}\left(z_{0}\right)
$$

where $\alpha_{\text {RayleighBackscatter }}$ is the Rayleigh backscatter coefficient. Please note that in this case a fully incoherent treatment is given to the scattered power $P_{S}$. This considerably simplifies the 
TABLE I

VALUES OF THE PARAMETERS USED IN THE THEORETICAL MODELING

\begin{tabular}{|c|c|c|c|}
\hline Symbol & Parameter & Value & Units \\
\hline$P_{R}^{+}, P_{R}^{-}$ & $\begin{array}{l}\text { Forward }(+) \text { and backward }(-) \text { input } \\
\text { Raman pump power }\end{array}$ & 600 & $\mathrm{~mW}$ \\
\hline$\alpha_{R}$ & $\begin{array}{l}\text { Fiber loss at the wavelength of the } \\
\text { Raman pump }(1455 \mathrm{~nm})\end{array}$ & 0.0553 & $\mathrm{~km}^{-1}$ \\
\hline$g_{R}$ & $\begin{array}{l}\text { Raman gain at the wavelength of the } \\
\phi \text { OTDR pump }\end{array}$ & 0.3 & $\mathrm{~W}^{-1} \mathrm{~km}^{-1}$ \\
\hline$P_{P}$ & $\phi \mathrm{OTDR}$ input pump peak power & 10 & $\mathrm{~mW}$ \\
\hline$\alpha_{P}$ & $\begin{array}{l}\text { Fiber loss at the wavelength of the } \\
\phi \mathrm{OTDR} \text { pump }(1550 \mathrm{~nm})\end{array}$ & 0.047 & $\mathrm{~km}^{-1}$ \\
\hline $\begin{array}{l}\alpha_{\text {Rayleigh }} \\
\text { Backscatter }\end{array}$ & Rayleigh Backscatter coefficient & -82 & $\mathrm{~dB} / \mathrm{m}$ \\
\hline
\end{tabular}

treatment of the equations and does not harm the main objective of our analysis, which is to determine the overall evolution of the back-scattered signal.

From that point $\left(z_{0}\right)$, the backscattered signal has to travel back to the detector, and therefore should also experience fiber losses and Raman gain. The evolution of the power of the $\phi$ OTDR signal correspondent to $z_{0}$ along the fiber can be obtained solving the equation:

$$
\frac{d P_{S}^{z_{0}}(z)}{d z}=+\alpha_{P} P_{S}^{z_{0}}(z)-g_{R} P_{S}^{z_{0}}(z) \cdot\left[P_{R}^{+}(z)+P_{R}^{-}(z)\right] .
$$

Considering the previous equations, we can now establish the evolution of the backscattered $\phi$ OTDR signal correspondent to $z_{0}$ for any position $z<z_{0}$. The evolution of the backscattered power will be given by

$$
\begin{aligned}
P_{S}^{z_{0}}\left(z \leq z_{0}\right)= & P_{S}^{z_{0}}\left(z_{0}\right) e^{\alpha_{P}\left(z-z_{0}\right)} \exp \left\{-\frac{g_{R}}{\alpha_{R}} P_{R}^{-}(L)\right. \\
& \times\left[e^{\alpha_{R}(z-L)}-e^{\alpha_{R}\left(z_{0}-L\right)}\right] \\
& \left.+\frac{g_{R}}{\alpha_{R}} P_{R}^{+}(0)\left[e^{-\alpha_{R} z}-e^{-\alpha_{R} z_{0}}\right]\right\} .
\end{aligned}
$$

At the detection point (input of the fiber), the $\phi$ OTDR signal correspondent to $z_{0}\left(P_{S}^{z}-0(0)\right)$ will be

$$
P_{S}^{z_{0}}(0)=\frac{P_{P}\left(z_{0}\right)}{P_{P}(0)} P_{S}^{z_{0}}\left(z_{0}\right)=\frac{P_{P}\left(z_{0}\right)^{2}}{P_{P}(0)} \alpha_{\text {RayleighBackscatter }} .
$$

The values of the parameters used in the theoretical modeling are presented in Table I.

\section{EXPERIMENTAL SETUP AND CHARACTERIZATION OF THE $\phi$ OTDR ASSISTED BY FIRST-ORDER RAMAN AMPLIFICATION}

\section{A. Setup}

The experimental setup used to characterize the $\phi \mathrm{OTDR}$ assisted by first-order Raman amplification is shown in Fig. 1. A laser diode (LD) with a linewidth of $1.6 \mathrm{MHz}$ emitting at $1548 \mathrm{~nm}$ was used as the master source. The used LD presents a high enough coherence for the experiment (LD coherence length

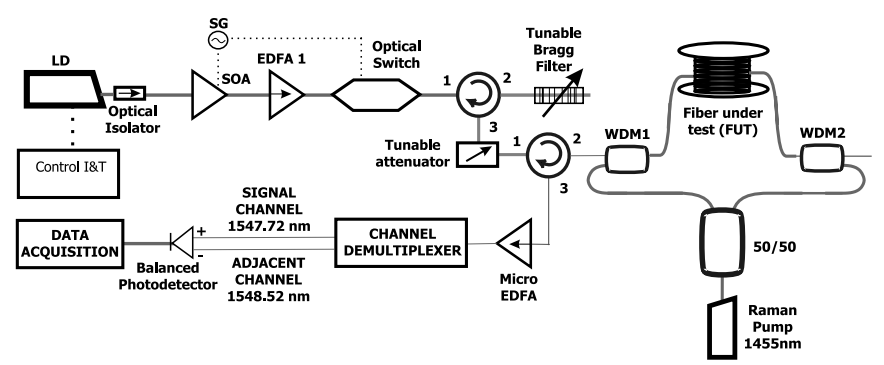

Fig. 1. Experimental setup: Acronyms are explained in the text.

$\approx 50 \mathrm{~m}$ while the pulse length in the fiber is $\approx 20 \mathrm{~m}$ ) but not too high, thus minimizing the generation of coherent noise due to the continuous leakage outside the pulses [5]. Also, it allows maintaining the simplicity of the scheme (the LD is driven by a standard current and temperature controller) and not presenting the technological challenges typically presented by lasers with sub-megahertz linewidths. An optical isolator was placed at the laser output to avoid disturbances in the laser coherence due to back-reflections.

An SOA, with rise/fall times in the order of $2.5 \mathrm{~ns}$, driven by a waveform signal generator (SG) was used to create $100 \mathrm{~ns}$ almost square pulses. Between the signal pulses, the SOA was negatively biased so as to enhance the ER of the delivered pulses. An ER of $>50 \mathrm{~dB}$ was achieved this way. An erbiumdoped fiber amplifier (EDFA) was used to boost the power of the $\phi$ OTDR input pulses and reach peak powers in the pulse of several hundred milliwatts. In order to minimize the effect of the ASE added by the EDFA, we inserted a tunable fiber Bragg grating (FBG) working in reflection. The spectral profile of the FBG is the typical spectrum of a $100 \%$ reflection FBG and its spectral width is $0.8 \mathrm{~nm}$. For the used settings, the leaked ASE power is below the leaked power of the master signal and therefore the ER decrease is not significant after the EDFA + FBG [22]. In addition to the FBG, an optical switch with rise/fall times of $200 \mathrm{~ns}$ and a typical ER of $25 \mathrm{~dB}$ was used after the EDFA. It was driven so as to have a sub-microsecond transmission window synchronized with the pulse. This system allows to further increase the ER, reducing the noise delivered into the fiber outside the pulse. As shown before [5], the ER has a very high impact on the SNR of the detected trace in normal $\phi$ OTDR operation. After that, light passed through an attenuator, which allowed varying the input pulse power in the fiber.

The fiber under test (110 km of SMF-28) (FUT), is connected to the common ports of two WDMs $(1450 / 1550 \mathrm{~nm})$ which had an isolation $>60 \mathrm{~dB}$ in the pass channel. The input pulse is launched into the FUT through the $1550 \mathrm{~nm}$ port of the WDM1. The Raman pump is a CW Raman Fiber Laser (RFL) emitting at $1455 \mathrm{~nm}$ with a RIN $<-110 \mathrm{dBc} / \mathrm{Hz}$. The power of this laser can be tuned up to $2 \mathrm{~W}$. The RFL beam is divided by a calibrated $50 / 50$ coupler in two beams and each beam is then coupled into the $1450 \mathrm{~nm}$ ports of the WDMs.

The signal back-reflected from the fiber is amplified (using a micro-EDFA) and then goes through a $100 \mathrm{GHz}$ channel 
demultiplexer which filters out the channel of the signal (centered at $1547.72 \mathrm{~nm}$ ) and an adjacent channel (centered at $1548.52 \mathrm{~nm}$ ), thus filtering out the ASE added by the microEDFA. The signal channel and the adjacent channel are then respectively coupled to the "+" and "-" port of a p-i-n balanced photodetector with amplification and $100 \mathrm{MHz}$ bandwidth. In this case the "+" port will receive the sum of signal and noise with a bandwidth of $100 \mathrm{GHz}$ and the "--" port will receive the noise of an adjacent channel with the same bandwidth. Since the optical paths leading to the "+" and "--" ports are the same, a maximum cancelation of the RIN is ensured. The detector's bandwidth should ideally be much larger than the pulse spectral bandwidth, since the $\phi$ OTDR traces exhibit high-contrast rapid oscillations. In our case the detector has a bandwidth roughly ten times higher than the pulse spectral bandwidth which is enough to adequately represent the process. The signal was then recorded using a high-speed digitizer.

\section{B. Noise Considerations}

It has been demonstrated that the noise suppression between the $\phi$ OTDR pulses in these types of systems is very important since it reduces the intra-band coherent noise of the setup. In the conventional settings, the use of an SOA allows achieving a high ER $(>50 \mathrm{~dB})$ and enhances the spectral purity of the laser spectrum in the active state due to spectral hole burning in the active material [5].

In the present setup, an optical switch was also used in order to further increase the ER of the $\phi$ OTDR pulses. This switch "windows" the input pulses and therefore avoids the leakage of a significant amount of noise between the pulses.

To illustrate the impact of the optical switch, the spectrum of the $\phi$ OTDR pulse after the FBG with the switch "on" and "off" is presented (see Fig. 2). The effect is clearly visible as a strong reduction of the ASE noise (as much as $22.5 \mathrm{~dB}$ reduction) was observed. This combination of SOA and optical switch results in a $\phi$ OTDR pulse with a very high ER and coherence, and a very low ASE noise delivered into the fiber.

Fig. 3(a) shows the spectrum reflected from the FUT (i.e., the $\phi$ OTDR signal) before the micro-EDFA. As expected, a background noise (with the spectral power distribution similar to the Raman gain) is observed due to the ASE introduced by the Raman amplification in the FUT. Further amplification in the micro-EDFA introduces more ASE noise. Even after spectral filtering, this ASE noise is comparable to the signal level, and therefore requires some kind of elimination strategy. Moreover, the effect of RIN is strongly manifested in this ASE noise.

The use of balanced detection among two adjacent channels (one with signal + ASE noise and the other with ASE noise only) allows suppressing the strong and very noisy dc component given by Raman ASE noise. The optical spectra of the signal channel $(1547.72 \mathrm{~nm})$ and the adjacent channel $(1548.52 \mathrm{~nm})$ received in the balanced detector is shown in Fig. 3(b). As we see, the amount of ASE noise subtracted is roughly equal in both channels. Furthermore, a power fluctuation in the Raman pump laser at a given time will affect the ASE level in both these channels similarly and therefore, by using the difference

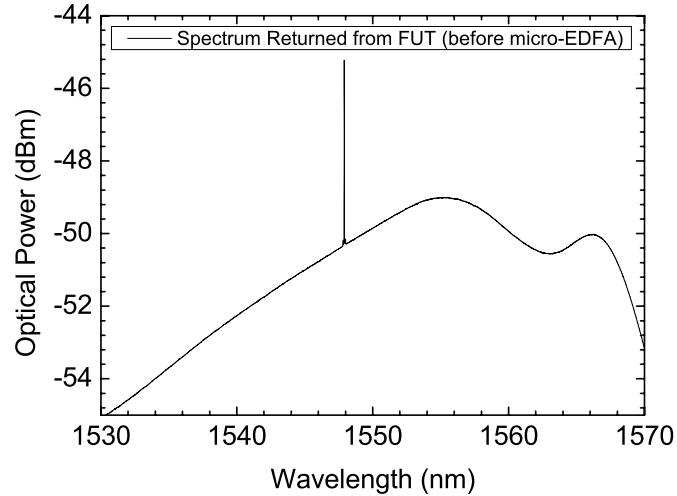

(a)

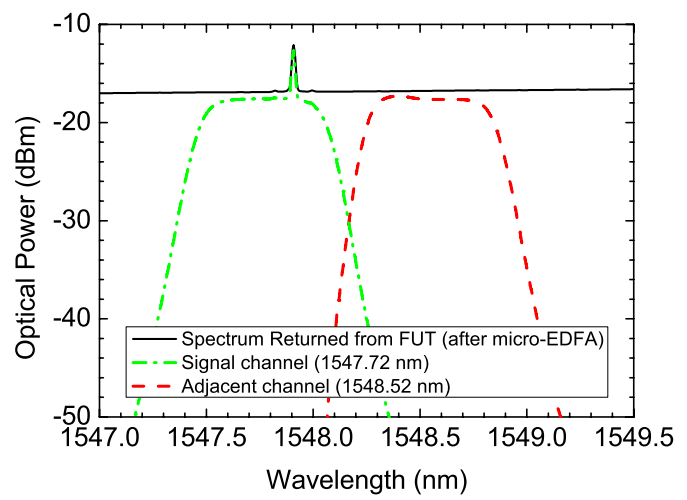

(b)

Fig. 2. Spectrum of the $\phi_{\mathrm{OTDR}}$ input pump pulses after passing the optical switch in the "on" versus "off" state. A clear increase of the ER $(\sim 22.5 \mathrm{~dB})$ was observed, mainly due to the reduction of the ASE introduced by the EDFA.

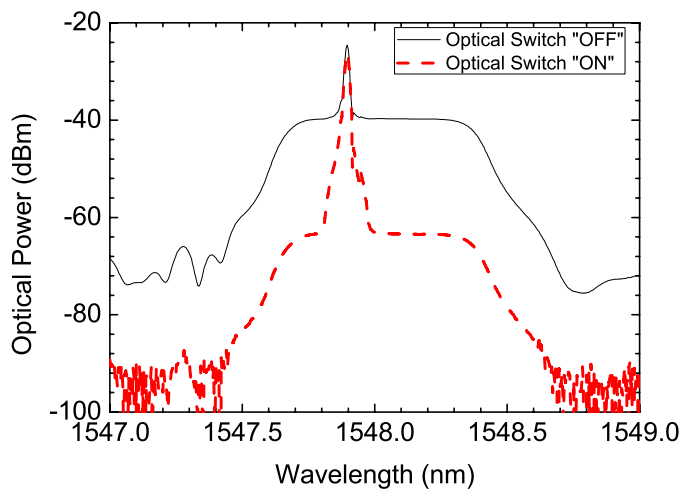

Fig. 3. (a) Optical spectrum returning from the FUT (before the micro-EDFA). (b) Optical spectrum returning from the FUT (after the micro-EDFA) and signals received in the "+" and "--" ports of the balanced detector (signal channel and adjacent channel).

between them, the effect of the RIN in the detected power along time is greatly diminished. Of course, for this noise cancellation to be efficient, the optical paths of the "+" and "-" signal in the detector have to be the same, as length mismatches are translated into a reduction of the bandwidth over which a perfect noise cancellation is achieved. For a bandwidth of $100 \mathrm{MHz}$, the detected power for each point is the average of the power which reaches the detector over $\sim 10$ ns (i.e. average of a light pulse 


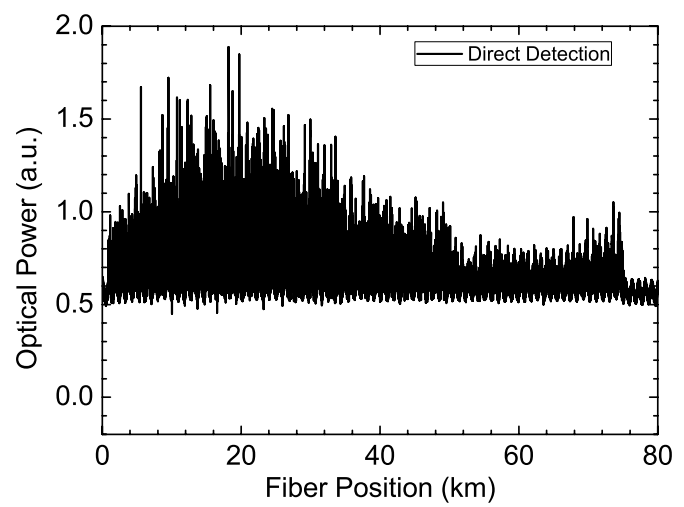

(a)

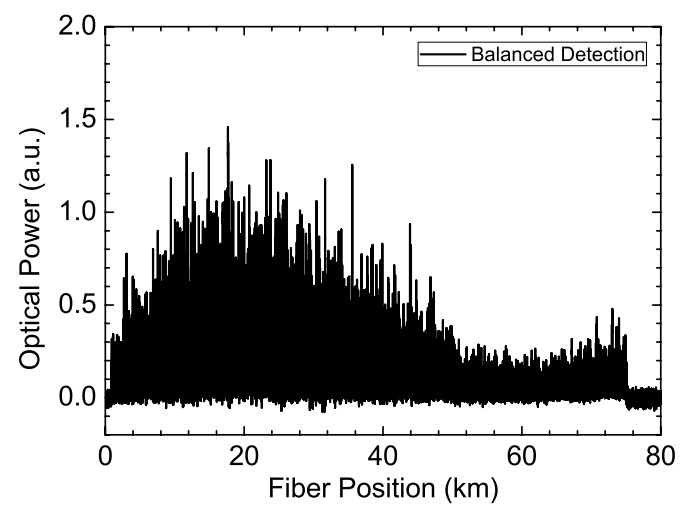

(b)

Fig. 4. Comparison of the RIN for (a) direct (b) balanced detection in a $75 \mathrm{~km}$ fiber span. The improvement is clearly visible as the periodical noise of the RIN is greatly reduced using balanced detection.

that covers $\sim 2$ meters of fiber) and therefore we can assure that noise cancellation will be effective with tolerances in the order of $1 \mathrm{~cm}$ in the optical paths of the "+" and "-" signal.

To illustrate the evidence of the improvement of balanced detection in comparison with direct detection, the two are compared in a situation in which the RIN is very high and its effects are noticeable (even in the time domain) in a $75 \mathrm{~km}$ fiber span (see Fig. 4). Fig. 4(a) shows the trace detected employing conventional detection and Fig. 4(b) shows the trace detected with our balanced scheme. The improvement is clearly visible as the periodical noise of the RIN shown using direct detection see Fig. 4(a) is greatly reduced using balanced detection see Fig. 4(b). Also, as expected, the dc component of the trace is eliminated.

In order to characterize the RIN transferred from the Raman pumps, a Raman pump power of $0.6 \mathrm{~W}$ was launched on each end of a $125 \mathrm{~km}$ FUT without input $\phi$ OTDR pulse. The signal returning from the fiber was then measured (photodetector + electrical spectrum analyzer, ESA) using direct and balanced detection. Results are presented in the electrical spectrum domain (see Fig. 5). It was observed that the direct detection presents a periodical structure with peaks at $140 \mathrm{kHz}$ (which are coincident with the period of $\sim 700 \mathrm{~m}$ of the RIN observed in Fig. 4(a). This period comes from the cavity length of the RFL. Strong low frequency $(<100 \mathrm{kHz})$ noise is also observed in the

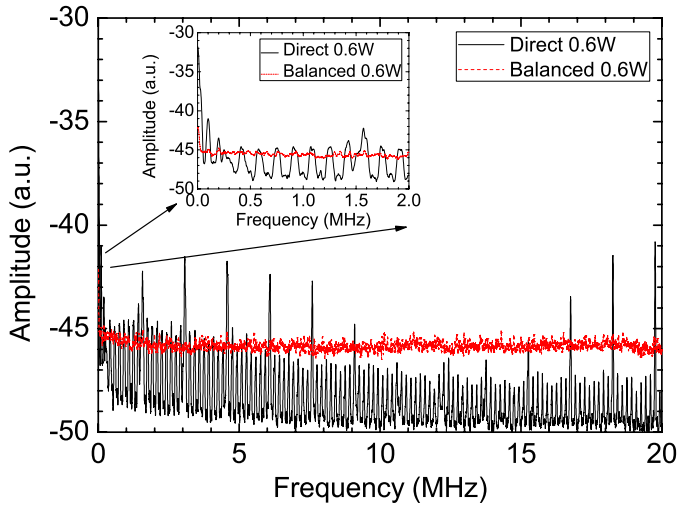

Fig. 5. RIN noise returned from $125 \mathrm{~km}$ FUT when a Raman pump power of $0.6 \mathrm{~W}$ launched on each end of the fiber without input $\phi_{\mathrm{OTD}} \mathrm{T}$ pump using balanced and direct detection. The direct detection presents a clear mode structure while the balanced detection only white noise is observed.

direct detection, which would greatly affect the comparison of equivalent points from different traces and therefore the vibration measurements. Using balanced detection only white noise is observed, proving the usefulness of this approach.

\section{IV. $\phi$ OTDR TRACES}

\section{A. $\phi O T D R$ Traces}

Fig. 6 shows the $\phi$ OTDR traces recorded for fiber spans of $110 \mathrm{~km}$ see Fig. 6(a) and $125 \mathrm{~km}$ see Fig. 6(b). As expected, the trace displays random oscillations and its amplitude varies with the $\phi$ OTDR pulse power evolution along the fiber. In both cases, the Raman pump power launched on each end of the fiber was $0.6 \mathrm{~W}$ (the same power which was used for the RIN characterization). As for the input $\phi$ OTDR pulse peak power estimated to be below $10 \mathrm{~mW}$ for both Fig. 4(a) and Fig. 4(b), it was chosen so as to ensure the best performance along all points of the fiber, i.e., the highest possible power before the appearance of nonlinearities (mainly MI, which leads to a fast decrease of the visibility) [7].

As it is clearly visible in Fig. 6(a), a considerable decrease of the amplitude of the trace oscillations at $\approx 98 \mathrm{~km}$ is observed. The same amplitude of oscillations decrease occurs at the same point in Fig. 6(b). This occurs due to a connector loss at this point (estimated in $3 \mathrm{~dB}$ ). It is important to mention that the point where the measurements were performed was immediately after the connector loss. Therefore, if the fiber was spliced in that point, this amplitude drop of the trace oscillations would not occur and better results could be obtained. However, as the vibrations were clearly measured, this loss was kept so as to prove the efficiency of our setup even in deteriorated conditions. It essentially proves that reliable vibration measurements can be performed well beyond the range of $100 \mathrm{~km}$ using this scheme.

The evolution of the Raman pump power and the $\phi$ OTDR pulse power given by the theoretical model (which includes the $3 \mathrm{~dB}$ loss due to the connector at $\sim 98 \mathrm{~km}$ ) is also presented and shown to have a good agreement with the experimental results. Although small differences can be expected for several reasons 


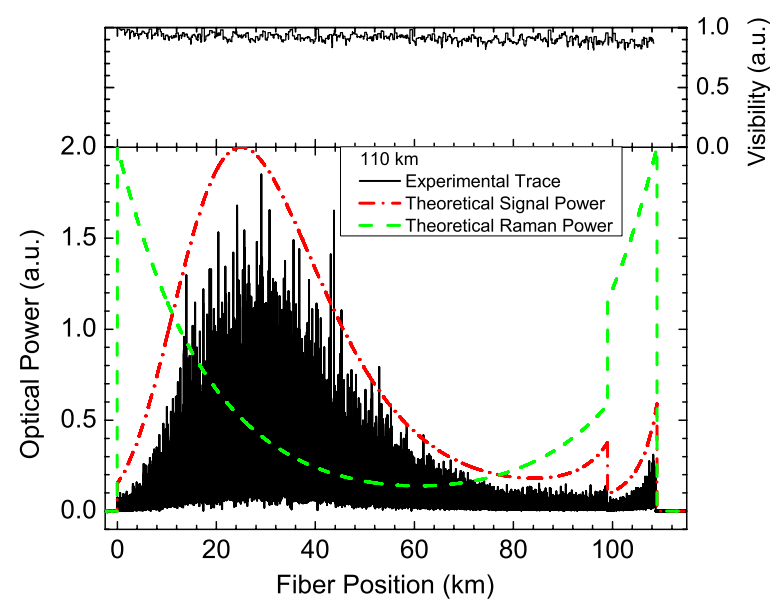

(a)

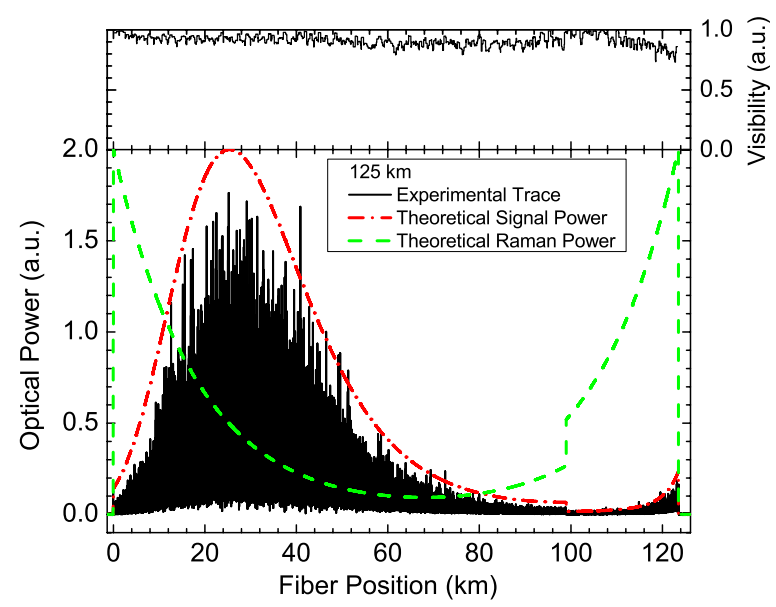

(b)

Fig. 6. $\phi_{\text {OTDR }}$ interference signal along the FUT of (a) $110 \mathrm{~km}$ and (b) $125 \mathrm{~km}$. The Raman pump power launched on each end of the fiber was $0.6 \mathrm{~W}$ and the $\phi_{\mathrm{OTDR}}$ pump power was chosen as the one ensuring the best performance in both cases. The theoretical modeling of the Raman pump power and $\phi_{\mathrm{OTDR}}$ pump power along the FUT is also presented. The top figure shows the visibility of the interference signal, computed as $\mathrm{V}=\left(\mathrm{T}_{\text {max }}-\mathrm{T}_{\text {min }}\right) /$ $\left(\mathrm{T}_{\max }+\mathrm{T}_{\min }\right)$, where $\mathrm{T}_{\max }$ and $\mathrm{T}_{\min }$ are the maximum and minimum values of the trace over a window of $500 \mathrm{~m}$.

(the fiber may present small inhomogeneities along the distance, some of the parameters in table I may show small errors) the evolution of the amplitude of the trace oscillations are shown to be coincident with the $\phi$ OTDR pulse power evolution along the fiber and the Raman pump power evolution is as qualitatively expected. The top figures show the visibility of the $\phi$ OTDR interference signal along the fiber, calculated as indicated in the figure caption. Despite the fact that the amplitude of the oscillations and average reflected power present large variations along the fiber, the variations of the contrast of the interference are much lower (the visibility remains close to 1 ). Since the visibility of the $\phi$ OTDR trace is not altered along the fiber, we can conclude that reliable vibration measurements can be performed at any position along the fiber if we can ensure that we can do vibration measurements in the position of worst signal level (around $\mathrm{km} 100$ in both cases). Thus, our vibration measurement tests are done in this position.
Although only bi-directional Raman amplification is shown in this article, the co-propagating and counter-propagating $\mathrm{Ra}$ man amplification configurations were also tested and observed to perform worse. The reason for this is that larger signal variations are obtained with these configurations. The bi-directional configuration ensures the minimum variation of signal power along the fiber, and therefore the best possible performance.

\section{B. Evolution of the Optical trace with the Raman Pump Power}

The evolution of the trace profile for increasing Raman pump powers $(0.6,0.9,1.2$ and $1.5 \mathrm{~W}$ of total Raman input power, inserted half on each side) using a FUT of $110 \mathrm{~km}$ is shown in Fig. 7. Again, the input $\phi$ OTDR pulse power was adjusted for each Raman pump power to achieve the best possible performance while avoiding nonlinearities. Thus, for increasing Raman pump powers, the input $\phi$ OTDR pulse power was decreased. It was observed that for Raman pump powers higher than $1.2 \mathrm{~W}(0.6 \mathrm{~W}$ on each side) the point with the lowest amplitude of trace oscillations was at the beginning of the fiber. This high pumping level is nevertheless, not used in practice, as the Raman noise is too large for performing reliable vibration measurements.

In agreement with equation (5), it can be seen that for increasing Raman pump powers the maximum of the input $\phi$ OTDR pulse power (when the Raman gain equals the fiber losses) occurs further into the fiber. This is the point in which Raman gain and linear fiber loss are equal. This condition will occur always for a certain level of Raman pump power, so for higher input Raman powers, this maximum comes obviously further inside the fiber.

\section{VibRation MEASUREMENTS}

Vibration measurements were carried out in a similar manner to the methodology described in [5]. For each point along the fiber, the optical power evolution as a function of time was obtained by measuring the equivalent point in consecutive traces and plotting the signal levels recorded for that point along the time. The sampling frequency at each position will therefore be the frequency at which the $\phi$ OTDR pulses are launched into the fiber, which is limited by the fiber length to be monitored.

Two FUT lengths were tested: $110 \mathrm{~km}$ and $125 \mathrm{~km}$. These consisted of two fiber spools of $50 \mathrm{~km}$ and one fiber spool of $10 / 25 \mathrm{~km}$ of SMF-28, all linked by optical connectors. The $\phi$ OTDR pulse repetition rate was $781 / 625 \mathrm{~Hz}$, which limits the maximum detectable frequency to $390.5 / 312.5 \mathrm{~Hz}$ (Nyquist theorem) and the theoretical maximum fiber span to be monitored to $131 / 163 \mathrm{~km}$.

The Raman pump power and input $\phi$ OTDR pulse power were the same as the ones used in the traces of Fig. 6(a) and (b). The point with the lowest amplitude of the trace oscillations, which in both cases was observed to be after the connector at $\approx 98 \mathrm{~km}$, was placed inside a $2 \mathrm{~m}$ long PVC tube with $0.08 \mathrm{~m}$ of diameter in which mechanical vibrations of controllable frequency were applied using a small vibration exciter with a maximum bare table acceleration of $736 \mathrm{~ms}^{-2}$ (with a $75 \mathrm{~g}$ mass attached). The 

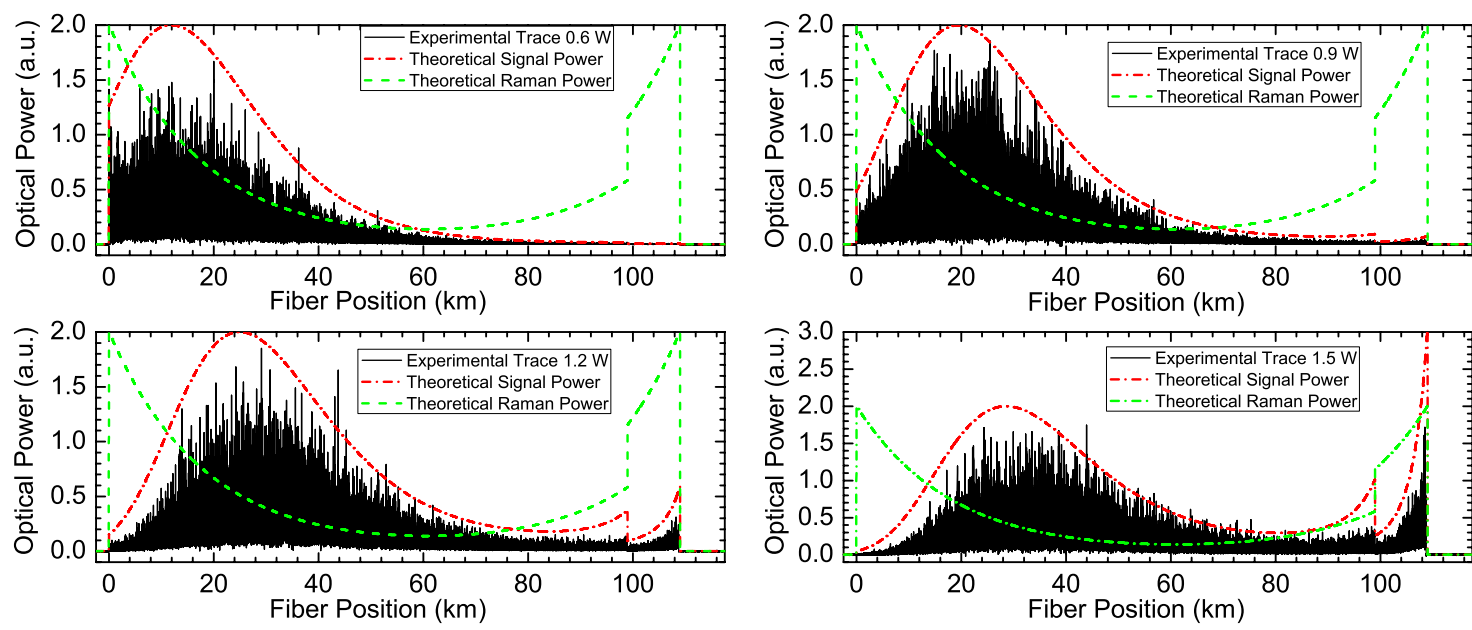

Fig. 7. Experimental and theoretical evolution of the trace profile for different Raman pump powers (total Raman input power), using an FUT of $110 \mathrm{~km}$. The $\phi_{\mathrm{OTDR}}$ pump power chosen for each Raman pump power was the one ensuring the best performance.

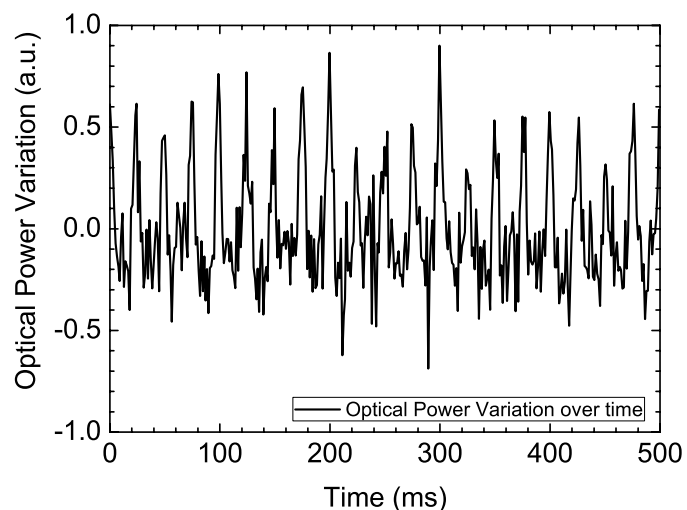

(a)

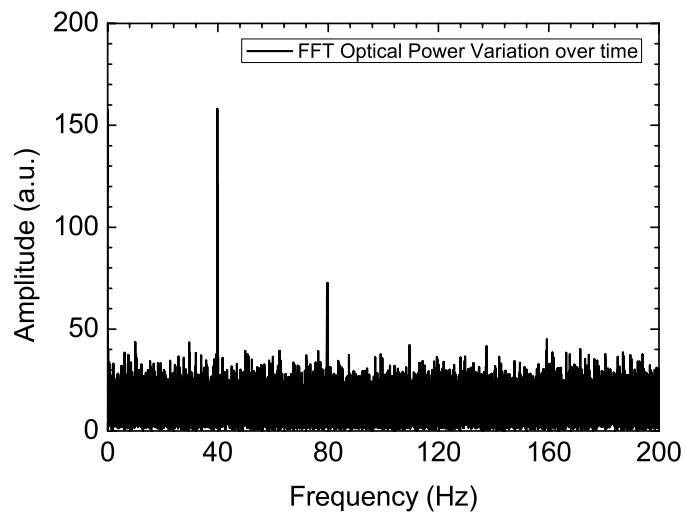

(b)

Fig. 8. (a) Optical power variation along time and (b) respective FFT of the $\phi_{\mathrm{OTDR}}$ signal in the fiber point with the minimum amplitude of the trace oscillations (km 98) of the $110 \mathrm{~km}$ FUT, using the same conditions of Fig. 6(a), for an applied vibration of $40 \mathrm{~Hz}$.

fiber was clamped outside the PVC tube in order to avoid the propagation of vibrations outside it.

Fig. 8(a) shows the optical power variation of the $\phi$ OTDR signal at the point inside the PVC tube when a vibration of
$40 \mathrm{~Hz}$ was applied to the tube. The trace is recorded using the same conditions of Fig. 6(a) $($ FUT $=110 \mathrm{~km})$ with no post-processing. A clear pattern with peaks synchronized with the applied frequency is observed. The Fast Fourier Transform (FFT) of the optical power variation is presented in Fig. 8(b). The $\phi \mathrm{OTDR}$ is observed to present a nonlinear response as a clear peak appears at $40 \mathrm{~Hz}$ followed by a smaller peak in the second harmonic $(80 \mathrm{~Hz})$. The optical power variations of the $\phi$ OTDR signal and respective FFTs of fiber points which were more than $10 \mathrm{~m}$ away from the PVC tube did not show sensitivity to the applied vibrations. This is in agreement with the expected resolution of $10 \mathrm{~m}$ (corresponding to a $100 \mathrm{~ns}$ pulse).

In order to test the limits of acquisition of the system, frequencies up to nearly the maximum detectable by the $\phi$ OTDR pulse repetition rate for each FUT were applied to the PVC tube. Fig. 9 presents the FFT spectra of the optical power variation recorded by the $\phi$ OTDR at the position of the shaker when the frequency applied to the PVC tube is raised from $10 \mathrm{~Hz}$ to $300 \mathrm{~Hz}$ using the FUT of $110 \mathrm{~km}$ see Fig. 9(a) and $10 \mathrm{~Hz}$ to $250 \mathrm{~Hz}$ using the FUT of $125 \mathrm{~km}$ see Fig. 9(b). In both cases, the measurement conditions are the same as those used in Fig. 6 for each FUT.

The recorded spectra showed clearly visible peaks in all the applied frequencies, although the amplitude of the detected frequencies was observed to have some instability. As expected, the measurements using the FUT of $110 \mathrm{~km}$ presented a higher SNR than when using the FUT of $125 \mathrm{~km}$.

For the maximum frequencies tested, the estimated displacement of the PVC tube is in the sub-millimeter range. It is important to stress that the point where the measurements are performed is immediately after a lossy connector, in the position with lowest sensitivity of the whole trace. Also, no post-processing was used in the presented data and therefore, to some extent, the performance of the sensor could be increased with the proper data treatment for specific applications. 


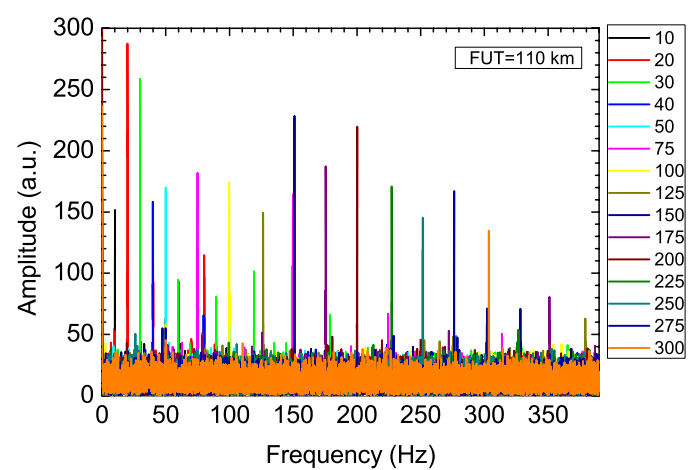

(a)

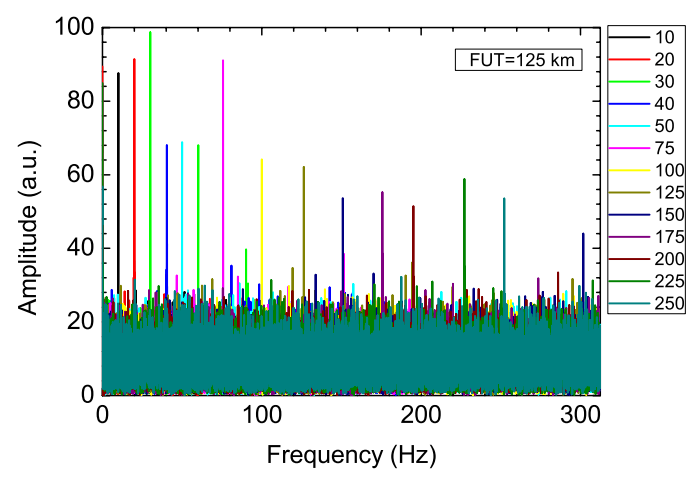

(b)

Fig. 9. FFT spectra of the optical power variation of the $\phi_{\mathrm{OTDR}}$ signal for consecutive traces in the fiber point with minimum amplitude of the trace oscillations (after km 98) of the (a) $110 \mathrm{~km}$ and (b) $125 \mathrm{~km}$ FUT for applied frequencies between $10 \mathrm{~Hz}$ and (a) $300 \mathrm{~Hz}$ and (b) $250 \mathrm{~Hz}$, using the same conditions of Fig. 6 for each FUT.

\section{CONCLUSION}

In this study, the authors present an experimental and theoretical description of the use of first-order Raman amplification to improve the performance of a $\phi$ OTDR when used for vibration measurements. The evolution of the $\phi$ OTDR signal along the fiber is shown to have a good agreement with the theoretical model for different Raman pump powers. The Raman amplification combined with the noise reduction provided by the use of balanced detection, an SOA and an optical switch allows to greatly increase the SNR and sensing range of the $\phi$ OTDR. Frequency measurements close to the limits set by the time of flight of light pulses are achieved this way in a range which, to our knowledge, is the highest for $\phi$ OTDR vibration sensing achieved so far. The sensor was able to detect vibrations of up to $250 / 300 \mathrm{~Hz}$ in a distance of $125 / 110 \mathrm{~km}$ with a resolution of $10 \mathrm{~m}$ and no post-processing. This sensor could be used in the monitoring of intrusions in large structures such as national borders or pipelines. The use of bi-directional Raman pumping is a clear drawback in this setup, as access to both fiber ends is required. However, unlike the case of Brillouin Optical Time Domain Analyzers, in this scheme there is no restriction in terms of having the two access points in the same place, as the Raman pumps on each side can be physically separated. In addition, depending on the application (for instance using a circular ge- ometry to monitor the perimeter of a military base), the use of bi-directional pumping may not be at all a limitation.

\section{REFERENCES}

[1] X. Bao and L. Chen, "Recent progress in distributed fiber optic sensors," Sensors-Basel, vol. 12, no. 7, pp. 8601-8639, 2012

[2] H. F. Taylor, and C. E. Lee"Apparatus and method for fiber optic intrusion sensing," U.S. Patent 5194847, Mar. 16, 1993.

[3] J. C. Juarez and H. F. Taylor, "Field test of a distributed fiber-optic intrusion sensor system for long perimeters," Appl Opt., vol. 46, no. 11, pp. 1968-1971, 2007.

[4] J. C. Juarez, E. W. Maier, K. N. Choi, and H. F. Taylor, "Distributed fiberoptic intrusion sensor system," J Lightw. Technol., vol. 23, no. 6, pp. 20812087, Jun. 2005

[5] H. F. Martins, S. Martin-Lopez, P. Corredera, M. L. Filograno, O. Frazao, and M. Gonzalez-Herraez, "Coherent noise reduction in high visibility phase sensitive optical time domain reflectometer for distributed sensing of ultrasonic waves," J. Lightw. Technol., vol. 31, no. 23, pp. 3631-3637, Dec. 2013

[6] Y. Koyamada, M. Imahama, K. Kubota, and K. Hogari, "Fiber-optic distributed strain and temperature sensing with very high measurand resolution over long range using coherent OTDR," J Lightw. Technol, vol. 27, no. 9, pp. 1142-1146, May 2009.

[7] H. F. Martins, S. Martin-Lopez, P. Corredera, P. Salgado, O. Frazão, and M. Gonzalez-Herraez, "Modulation instability-induced fading in phasesensitive optical time-domain reflectometry," Opt. Lett., vol. 38, no. 6, pp. 872-874, 2013.

[8] H. Cho and C. J. Lissenden, "Structural health monitoring of fatigue crack growth in plate structures with ultrasonic guided waves," Struct. Health Monit., vol. 11, no. 4, pp. 393-404, 2012.

[9] W. Jie, J. Xin-Hong, and R. Yun-Jiang, "Phase-sensitive optical timedomain reflectometer based on bi-directional Raman amplification," Acta Phys. Sin, vol. 62, no. 4, p. 044212, 2013.

[10] Z. Qin, L. Chen, and X. Bao, "Wavelet denoising method for improving detection performance of distributed vibration sensor," IEEE Photon. Technol. Lett., vol. 24, no. 7, pp. 542-544, Apr. 2012.

[11] J. Hegarty, N. A. Olsson, and L. Goldner, "CW pumped raman preamplifier in a $45 \mathrm{~km}$-long fibre transmission system operating at $1.5 \mu \mathrm{m}$ and $1 \mathrm{Gbit} / \mathrm{s}, "$ Electron. Lett., vol. 21, no. 7, pp. 290-292, 1985.

[12] Y. Aoki, "Properties of fiber Raman amplifiers and their applicability to digital optical communication systems," J. Lightw. Technol., vol. 6, no. 7, pp. 1225-1239, Jul. 1988.

[13] P. B. Hansen, L. Eskildsen, S. G. Grubb, A. J. Stentz, T. A. Strasser, J. Judkins, J. J. DeMarco, R. Pedrazzani, and D. J. DiGiovanni, "Capacity upgrades of transmission systems by raman amplification," IEEE Photon. Tech. L., vol. 9, no. 2, pp. 262-264, Feb. 1997.

[14] S. K. Turitsyn, J. D. Ania-Castanon, S. A. Babin, V. Karalekas, P. Harper D. Churkin, S. I. Kablukov, A. E. El-Taher, E. V. Podivilov, and V. K. Mezentsev, "270-km ultralong Raman fiber laser," Phys. Rev. Lett., vol. 103, no. 13, p. 133901, 2009.

[15] M. Alahbabi, Y. Cho, and T. Newson, "150-km-range distributed temperature sensor based on coherent detection of spontaneous Brillouin backscatter and in-line Raman amplification," J. Opt. Soc. Amer. B, vol. 22, no. 6 , pp. 1321-1324, 2005.

[16] F. Rodriguez-Barrios, S. Martin-Lopez, A. Carrasco-Sanz, P. Corredera J. D. Ania-Castanon, L. Thevenaz, and M. Gonzalez-Herraez, "Distributed brillouin fiber sensor assisted by first-order Raman amplification," $J$. Lightw. Technol., vol. 28, no. 15, pp. 2162-2172, Aug. 2010.

[17] H. F. Martins, M. B. Marques, and O. Frazao, "300 km-ultralong raman fiber lasers using a distributed mirror for sensing applications," Opt. Exp., vol. 19, no. 19, pp. 18149-18154, 2011.

[18] J. D. Ania-Castanon, T. J. Ellingham, R. Ibbotson, X. Chen, L. Zhang, and S. K. Turitsyn, "Ultralong raman fiber lasers as virtually lossless optical media," Phys. Rev. Lett., vol. 96, no. 2, p. 023902, 2006.

[19] S. Martin-Lopez, M. Alcon-Camas, F. Rodriguez-Barrios, P. Corredera, J. D. Ania-Castanon, L. Thevenaz, and M. Gonzalez-Herraez, "Brillouin optical time-domain analysis assisted by second-order Raman amplification," Opt. Exp., vol. 18, no. 18, pp. 18769-18778, 2010.

[20] B. Bristiel, S. Jiang, P. Gallion, and E. Pincemin, "New model of noise figure and RIN transfer in fiber Raman amplifiers," IEEE Photon. Tech. L., vol. 18, no. 8, pp. 980-982, Apr. 2006. 
[21] J. Nuno, M. Alcon-Camas, and J. Ania-Castanon, "RIN transfer in random distributed feedback fiber lasers," Opt. Exp., vol. 20, no. 24, pp. 2737627381, 2012.

[22] D. M. Baney, P. Gallion, and R. S. Tucker, "Theory and measurement techniques for the noise figure of optical amplifiers," Opt. Fiber Technol., vol. 6 , no. 2, pp. 122-154, 2000.

Hugo F. Martins received the B.Sc. and M.Sc. degrees in physics from the University of Porto, Porto, Portugal, in 2009 and 2011, respectively. He is currently working toward the Ph.D. degree under jointly-awarded program in the University of Porto, Porto, Portugal and the University of Alcalá, Madrid, Spain.

During his M.Sc. dissertation work, he was with INESC Porto in the development of fiber sensing applications using cooperative Rayleigh-Raman scattering. His current research interests include studying the possibility of using ultralong fiber lasers based on distributed Rayleigh mirrors to obtain a new all-fiber secure key distribution system (while working in Porto, with INESC TEC) and distributed fiber sensors, mainly the use of phase-sensitive optical time domain reflectometry for distributed vibration detection (while working in the University of Alcalá).

Sonia Martín-López received the Ph.D. degree from the Universidad Complutense de Madrid, Madrid, Spain, in May 2006. The topic of her doctoral dissertation was on experimental and theoretical understanding of continuous-wave pumped supercontinuum generation in optical fibers. She had a pre-doctoral stay in the Nanophotonics and Metrology Laboratory, Ecole Polytechnique Federale de Lausanne, Switzerland. She has been engaged as a Postdoctoral Researcher in the Applied Physics Institute and in the Optics Institute at the Spanish Council for Research during six years. She is currently a Postdoctoral Researcher in the Photonics Engineering Group, University of Alcala, Madrid, Spain. She is author or coauthor of more than 100 papers in international refereed journals and conference contributions. Her current research interests include nonlinear fiber optics and distributed optical fiber sensors.

Pedro Corredera received the B.Sc. and Ph.D. degrees in physics from the University of Salamanca, Salamanca, Spain, in 1985 and 1989, respectively.

In 1989, he joined the Institute of Optics, Spanish National Research Council, where he works on IR radiometry and optical fibres metrology. In 1990-1991, he joined at National Physical Laboratory (NPL, Teddington/UK), working in Cryogenic Radiometry and optical fibre metrology. From 1996-2010, he joined the Institute of Applied Physics (IFA-CSIC) and he created a research group in Optical Communications Technologies (GiTCO) with three lines of work: optical fibers metrology, nonlinear properties of optical fibers, and applications and optical fiber sensors. In 2010, he rejoined the Institute of Optics (IO-CSIC) in the group "nonlinear dynamics and fiber optics." He published more than 60 articles in scientific and technical journals and 100 contributions at international conferences. His current research interests include fiber-optic measurements, optical fiber sensors, nonlinear fiber optics, and IR radiometry and detection.

Dr. Corredera is a member of the Spanish Society of Optics, the Optical Society American (OSA), and of the European Optical Society (EOS).
Massimo L. Filograno received the Laurea (5 year program) degree in electronic engineering from the Polytechnic of Bari, Bari, Italy, in 2009, discussing the thesis entitled "Real Time Monitoring of railway infrastructures using technology based on fiber Bragg grating." He has been working with the GRIFO (Group of Photonics Engineering of the University of Alcala, Madrid) since September 2008, and with CSIC (the Spanish National Research Council) since September 2010, toward the Ph.D. degree of the Information Engineering Department, University of Alcala. His main research interests include optical fiber sensors applied to high-speed train systems.

Orlando Frazão received the Graduation degree in physics engineering (optoelectronics and electronics) from the University of Aveiro, Aveiro, Portugal, in 1999, and the Ph. D. degree from the University of Porto, Porto, Portugal, in 2009 , on optical fiber sensors based on interferometry and nonlinear effects

From 1997 to 1998, he was with the Institute of Telecommunications, Aveiro, Portugal, with a scholarship in the European Project UPGRADE (High Bitrate $1300 \mathrm{~nm}$ Upgrade of the European Standard Single Mode Network.). He is currently a Senior Researcher and a Head of the Group of optical fiber sensor for Physical measurements at Optoelectronics and Electronic Systems Unit, INESC Porto. His current research interests included optical fiber sensors and optical communications. He has conducted several National research projects in optical sensing and optical communications and has also participated in an European project (NEXTGENPCF-Next Generation Photonic Crystal). He has several bilateral cooperation projects between France (Xlim, Université de Nice Sophie Antipolis), Spain (Universidad Carlos III de Madrid), Brasil Universidade Federal do Para (UFPA), and Poland (WRUT). He has published about 120 articles in peer-reviewed journals, more than 220 papers in international and National conference proceedings, and authored 12 patents. He has been a Reviewer for several international journals of the following societies: IEEE, OSA, Elsevier, IOP and others. He has participated as part of the organizer committee of several conferences.

Dr. Orlando Frazão received two international Scientific awards. He is also a Senior member of the SPIE.

Miguel González-Herráez received the M.Eng. and D.Eng. degrees from the Polytechnic University of Madrid, Madrid, Spain, in 2000 and 2004, respectively.

While working toward the D.Eng. degree, he worked first as a Research Assistant and then as a Postdoctoral Fellow in the Applied Physics Institute, Spanish Council for Research, Madrid, Spain, and had several long stays in the Nanophotonics and Metrology Laboratory, Ecole Polytechnique Federale de Lausanne, Switzerland. In October 2004, he was appointed as an Assistant Professor in the Department of Electronics, University of Alcalá, Madrid, Spain, where he was promoted to an Associate Professor in June 2006. He is the author or coauthor of more than 130 papers in international refereed journals and conference contributions and has given several invited talks at international conferences. His research interests include the wide field of nonlinear interactions in optical fibers.

Dr. González-Herráez has received several important recognitions to his research career, including the European Research Council Starting Grant and the "Agustin de Betancourt" prize of the Spanish Royal Academy of Engineering. $\mathrm{He}$ is also a Senior Member of the Optical Society of America. 